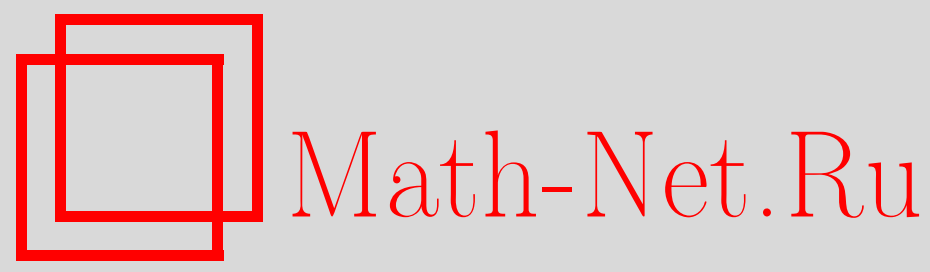

К. И. Пономарев, Параметрическая модель вкрапления и ее статистический анализ, Дискрет. матем., 2009, том 21, выпуск 4, 148-157

DOI: https://doi.org/10.4213/dm1079

Использование Общероссийского математического портала Math-Net.Ru подразумевает, что вы прочитали и согласны с пользовательским соглашением http://www . mathnet.ru/rus/agreement

Параметры загрузки:

IP: 18.209 .158 .208

26 апреля 2023 г., 11:58:39 


\title{
Параметрическая модель вкрапления и ее статистический анализ
}

\author{
() 2009 г. К. И. Пономарев
}

\begin{abstract}
Анализируется модель вкрапления передаваемой закрытой информации, когда в исходной бернуллиевской последовательности каждый элемент с определенной вероятностью заменяется на альтернативный бернуллиевский знак. Определяются условия на параметры модели, гарантирующие надежность сокрытия факта вкрапления. С аналогичных позиций проанализирована также полиномиальная модель.
\end{abstract}

\section{1. Введение}

Стенографический термин вкрапление означает замену некоторых символов передаваемой по каналу связи открытой информации альтернативными символами, несущими маскируемую конфиденциальную информацию. Основной проблемой стеганографии является такая организация процесса вкрапления, при которой обеспечивается гарантированный уровень надежности сокрытия от противника самого факта вкрапления. С математической точки зрения здесь речь идет о различении по наблюдаемому сообщению гипотез $H_{0}$ и $H_{1}$ об отсутствии и наличии вкраплений соответственно. При этом для передающей стороны наибольший интерес представляет определение условий, при которых эти гипотезы статистически неразличимы. Решение этих задач существенно зависит от соответствующих математических моделей вкрапления, и разработка таких моделей и их статистический анализ являются актуальной проблематикой современной стеганографии.

В предыдущей работе автора [2] была рассмотрена одна простейшая модель вкрапления, когда в исходную бернуллиевскую последовательность $\xi=\left(\xi_{1}, \ldots, \xi_{n}\right)$ независимо вкрапляется некоторое фиксированной число $k$ альтернативных бернуллиевских элементов. Здесь мы рассматриваем другой вариант вкрапления, когда каждый элемент последовательности $\bar{\xi}$ с некоторой вероятностью $\theta>0$ заменяется альтернативным бернуллиевским знаком, тем самым число $k$ вкрапляемых знаков в этом случае случайно. Как и в [2], внимание акцентируется на выявлении условий на параметры модели, обеспечивающих асимптотическую при $n \rightarrow \infty$ неразличимость гипотез $H_{0}: \theta=0$ (вкрапления отсутствуют) и $H_{1}: \theta>0$ (наличие вкраплений). Оказывается, что выводы для обеих моделей в среднем аналогичны. Подобный анализ проведен для полиномиальной модели, когда элементы последовательности $\bar{\xi}$ принимают значения в произвольном конечном алфавите. 


\section{2. Модель вкрапления и достаточная статистика для нее}

Достаточно общая модель вкрапления может выглядеть так: имеются три независимые последовательности из независимых одинаково распределенных случайных величин

$$
\begin{aligned}
\bar{\xi}_{0} & =\left(\xi_{01}, \xi_{02}, \ldots, \xi_{0 n}\right), \\
\bar{\xi}_{1} & =\left(\xi_{11}, \xi_{12}, \ldots, \xi_{1 n}\right), \\
\bar{\xi} & =\left(\delta_{1}, \delta_{2}, \ldots, \delta_{n}\right),
\end{aligned}
$$

где

$$
\begin{gathered}
\mathscr{L}\left(\xi_{01}\right)=F_{0}, \quad \mathscr{L}\left(\xi_{11}\right)=F_{1}, \\
\mathbf{P}\left(\delta_{1}=1\right)=1-\mathbf{P}\left(\delta_{1}=0\right)=\theta>0 .
\end{gathered}
$$

а наблюдается последовательность $\bar{\xi}=\left(\xi_{1}, \ldots, \xi_{n}\right)$, где

$$
\xi_{i}=I\left(\delta_{i}=1\right) \xi_{0 i}+I\left(\delta_{i}=1\right) \xi_{1 i}, \quad i=1, \ldots, n,
$$

здесь $I(A)$ - индикатор события $A$.

Последовательность $\bar{\xi}_{0}$ интерпретируется как исходное открытое сообщение, $\bar{\xi}_{1}-$ как вкрапляемая в $\bar{\xi}_{0}$ конфиденциальная информация и $\bar{\delta}-$ как механизм вкрапления. Задача состоит в такой организации процесса вкрапления, чтобы по наблюдению последовательности $\bar{\xi}$ противник не мог обнаружить факт вкрапления, то есть различить гипотезы $H_{0}: \theta=0$ и $H_{1}: \theta>0$.

Возможны различные конкретизации и усложнения описанной модели, и далее мы будем рассматривать один ее частный (близкий к стеганографической практике) вариант, когда $\bar{\xi}_{0}$ и $\bar{\xi}_{1}-$ бернуллиевские последовательности.

Итак, пусть

$$
\begin{gathered}
\mathscr{L}\left(\xi_{01}\right)=\operatorname{Bi}\left(1, p_{0}\right), \quad \mathscr{L}\left(\xi_{11}\right)=\operatorname{Bi}\left(1, p_{1}\right), \\
\Delta=p_{0}-p_{1}>0 ;
\end{gathered}
$$

(здесь и ниже $\operatorname{Bi}(n, p)$ - биномиальное распределение с параметрами $(n, p))$. Тогда наблюдаемая последовательность $\bar{\xi}=\left(\xi_{1}, \ldots, \xi_{n}\right)$ также является бернуллиевской и

$$
p_{\theta}=\mathbf{P}_{\theta}\left(\xi_{i}=1\right)=(1-\theta) p_{0}+\theta p_{1}=p_{0}-\theta \Delta \in\left[p_{1}, p_{0}\right] \text {. }
$$

Известно (см., например, [1]), что для бернуллиевской модели $\mathrm{Bi}\left(1, p_{\theta}\right)$ полной достаточной статистикой является наблюдаемое число единиц

$$
\rho_{n}=\sum_{l=1}^{n} \xi_{i},
$$

при этом

$$
\mathscr{L}\left(\rho_{n}\right)=\operatorname{Bi}\left(n, p_{\theta}\right) .
$$

Таким образом, в рассматриваемом случае все сводится к получению статистических выводов о параметре $\theta$ в модели, определяемой (1) и (2). Ниже решаются две основные задачи:

(1) проверка гипотезы $H_{0}: \theta=0$ об отсутствии вкраплений) при альтернативе $H_{1}: \theta>0$ о наличии вкраплений;

(2) оценивание интенсивности вкраплений $\theta$ (при справедливости гипотезы $H_{1}$ ). 
Замечание 1. Величина $p_{1}=\mathbf{P}\left(\xi_{11}=1\right)$, а тем самым и $\Delta=p_{0}-p_{1}$, вообще говоря, также должна рассматриваться как неизвестный параметр модели, но поскольку $\theta$ и $\Delta$ присутствуют в (1) в виде произведения, фактически рассматриваемая модель зависит лишь от одного параметра $\theta^{\prime}=\theta \Delta$. В дальнейшем мультипликативный параметр $\Delta$ можно считать известным и все рассуждения вести в терминах лишь параметра $\theta$.

\section{3. Различение гипотез}

Пусть $H_{\theta}$ означает простую гипотезу о наличии вкраплений с заданной интенсивностью вкраплений $\theta>0$. Запишем отношение правдоподобия $L_{\theta} / L_{0}$ в задаче $\left(H_{0}, H_{\theta}\right)$ различения гипотез $H_{0}$ и $H_{\theta}$ :

$$
\frac{L_{\theta}}{L_{0}}=\frac{p_{\theta}^{\rho_{n}} q_{\theta}^{n-\rho_{n}}}{p_{0}^{\rho_{n}} q_{0}^{n-\rho_{n}}}=\varphi^{\rho_{n}}(\theta)\left(\frac{q_{\theta}}{q_{0}}\right)^{n},
$$

где

$$
q_{\theta}=1-p_{\theta}, \quad \varphi(\theta)=\frac{q_{0} p_{\theta}}{p_{0} q_{\theta}} .
$$

Здесь функция $\varphi(\theta), \theta \in[0,1]$, обладает следующими свойствами:

$$
\begin{aligned}
\varphi(0) & =1, \\
\varphi(1) & =\frac{q_{0} p_{1}}{p_{0} q_{1}}=\frac{\left(1-p_{0}\right) p_{1}}{p_{0}\left(1-p_{1}\right)}, \\
\varphi^{\prime}(\theta) & =\frac{q_{0}}{p_{0}} \frac{-\left(q_{0}+\theta \Delta\right)-\left(p_{0}-\theta \Delta\right)}{q_{\theta}^{2}}=-\frac{q_{0} \Delta}{p_{0} q_{\theta}^{2}}<0 .
\end{aligned}
$$

Таким образом, $\varphi(\theta)<1$ при $\theta>0$, и поэтому отношение правдоподобия (3) монотонно убывает при возрастании $\rho_{n}$.

Итак, рассматриваемая модель имеет монотонное отношение правдоподобия, и поэтому (см. раздел 4.4.1 в [1]) в задаче $\left(H_{0}, H_{1}\right)$ проверки простой гипотезы $H_{0}$ при сложной односторонней альтернативе $H_{1}: \theta>0$ существует равномерно наиболее мощный критерий, который совпадает с критерием Неймана-Пирсона в задаче $\left(H_{0}, H_{\theta}\right)$ с простой альтернативой $H_{\theta}$.

Последний же критерий задается критической областью вида $L_{\theta} / L_{0} \geqslant c$ (см. раздел 4.2 .3 в [1]). В нашем случае это эквивалентно условию $\rho_{n} \leqslant t_{\alpha}$, где критическая граница $t_{\alpha}$ определяется заданием уровня значимости $\alpha=\mathbf{P}\left(H_{\theta} \mid H_{0}\right)$ :

$$
\alpha=\mathbf{P}_{0}\left(\rho \leqslant t_{\alpha}\right)=\sum_{j \leqslant t_{a}} b_{j}\left(n, p_{0}\right)=B\left(t_{\alpha} ; n, p_{0}\right),
$$

где

$$
b_{j}(n, p)=\left(\begin{array}{l}
n \\
j
\end{array}\right) p^{j} q^{n-j}, \quad j=0,1, \ldots, n,
$$

и $B\left(x ; n, p_{0}\right)$ - функция биномиального распределения $\operatorname{Bi}\left(n, p_{0}\right)$.

В силу ступенчатости функции распределения

$$
B\left(x ; n, p_{0}\right)=\mathbf{P}\left(\rho_{n} \leqslant x\right),
$$


уравнение (4) при заданных значениях параметров $n, p_{0}$ и $\alpha$ может не иметь решения. Поэтому в общем случае нужно поступать так, как описано в разделе 4.2 в [1]. При заданном $\alpha$ определим целое $t_{\alpha}$ условиями

$$
\alpha^{\prime \prime}=B\left(t_{\alpha}-1 ; n, p_{0}\right)<\alpha \leqslant B\left(t_{\alpha} ; n, p_{0}\right)=\alpha^{\prime} .
$$

Если здесь имеет место равенство $\alpha=\alpha^{\prime}$, то критерий задается критической областью $X_{1 \alpha}^{*}$, которая не зависит от альтернативы $H_{\theta}$ и поэтому определяет равномерно наиболее мощный критерий уровня значимости $\alpha$ в задаче $\left(H_{0}, H_{1}\right)$. Функция мощности вычисляется по формуле

$$
W\left(X_{1 \alpha}^{*} ; \theta\right)=\mathbf{P}_{\theta}\left(\rho_{n} \leqslant t_{\alpha}\right)=B\left(t_{\alpha} ; n, p_{\theta}\right)
$$

Если же в (5) выполняется неравенство $\alpha<\alpha^{\prime}$, то соответствующий критерий является рандомизированным с критической функцией

$$
\varphi_{\alpha}^{*}= \begin{cases}1, & \rho_{n} \leqslant t_{\alpha}-1, \\ \frac{\alpha-\alpha^{\prime \prime}}{b_{0}}, & \rho_{n}=t_{\alpha}, \\ 0, & \rho_{n} \geqslant t_{\alpha} \geqslant t_{\alpha}+1,\end{cases}
$$

где $b_{0}=b_{t_{\alpha}}\left(n, p_{0}\right)$ (гипотеза $H_{0}$ отвергается, если $\rho_{n} \leqslant t_{\alpha}-1$, и применяется, если $\rho_{n} \geqslant t_{\alpha}+1$; если $\rho_{n}=t_{\alpha}$, то $H_{0}$ отвергается с вероятностью $\left(\alpha-\alpha^{\prime \prime}\right) / b_{0}$ и принимается с дополнительной вероятностью $\left.\left(\alpha^{\prime}-\alpha\right) / b_{0}\right)$.

Мощность такого критерия при альтернативе $H_{\theta}$ равна

$$
W\left(\varphi^{*}-\alpha ; \theta\right)=\mathbf{E}_{\theta} \varphi_{\alpha}^{*}\left(\rho_{n}\right)=B\left(t_{\alpha}-1 ; n, p_{\theta}\right)+\left(\alpha-\alpha^{*}\right)\left(\frac{p_{\theta}}{p_{0}}\right)^{t_{\alpha}}\left(\frac{q_{\theta}}{q_{0}}\right)^{n-t_{\alpha}}
$$

На практике обычно имеют дело с большими выборками, поэтому вместо точных (и громоздких) формул (4)-(6) удобно использовать их асимптотический при $n \rightarrow \infty$ вариант. В рассматриваемом случае из (1)-(2) следует, что при любом $\theta$ при $n \rightarrow \infty$

$$
\mathscr{L}_{\theta}\left(\rho_{n}\right) \sim N\left(n p_{\theta}, n p_{\theta} q_{\theta}\right),
$$

поэтому критическую границу $t_{\alpha}=t_{\alpha}(n)$ в (4) следует выбрать в виде

$$
t_{\alpha}(n)=n p_{0}+\zeta_{\alpha} \sqrt{n p_{0} q_{0}},
$$

где $\zeta_{\alpha}$ есть $\alpha$-квантиль стандартного нормального распределения $N(0,1)$ :

$$
\Phi\left(\zeta_{\alpha}\right)=\frac{1}{\sqrt{2 \pi}} \int_{-\infty}^{\zeta_{\alpha}} e^{-x^{2} / 2} d x=\alpha .
$$

Для мощности $W_{n}(\theta)$ в этом случае из (6) следует, что

$$
\begin{aligned}
W_{n}(\theta) & =\mathbf{P}_{\theta}\left(\frac{\rho_{n}-n p_{\theta}}{\sqrt{n p_{\theta} q_{\theta}}} \leqslant \sqrt{n} \frac{\theta \Delta}{\sqrt{p_{\theta}} q_{\theta}}+\zeta_{\alpha} \sqrt{\frac{p_{0} q_{0}}{p_{\theta} q-\theta}}\right) \\
& \rightarrow \begin{cases}\Phi\left(t / \sqrt{p_{0} q_{0}}+\zeta_{\alpha}\right), & \text { если } \sqrt{n} \theta \Delta \rightarrow t \geqslant 0, \\
1, & \text { если } \sqrt{n} \theta \Delta \rightarrow \infty .\end{cases}
\end{aligned}
$$

Таким образом, справедливо следующее утверждение. 
Теорема 1. В задаче проверки гипотезы $H_{0}: \theta=0$ при альтернативе $H_{1}: \theta>0$ существует равномерно наиболее мощный критерий, задаваемый при $n \rightarrow \infty$ критической областью $\left\{\rho_{n} \leqslant t_{\alpha}(n)\right\}$, где при заданном уровне значимости $\alpha$ граница $t_{\alpha}(n)$ определяется соотношением (8); мощчность этого критерия асимптотически имеет вид (9).

Из этой теоремы следует, что гипотезы $H_{0}$ и $H_{1}$ асимптотически неразличимы, если выполняется условие

$$
\sqrt{n} \theta \Delta \rightarrow 0
$$

Это и есть искомое условие для организации (в рамках рассматриваемой модели) процесса вкрапления скрытой информации в передаваемое сообщение: оно означает, что параметры $p_{1}=p_{0}-\Delta$ и $\theta$ должны быть такими, чтобы произведение $\theta \Delta$ было мало по сравнению с $1 / \sqrt{n}$ (при больших $n$ ).

В свою очередь, это условие может быть обеспечено двумя способами:

(1) $\theta \sqrt{n} \rightarrow 0$, то есть $\theta=\theta_{n}=o(1 / \sqrt{n})$, тогда параметр $p_{1}$ может быть любым (напомним, что $p_{1}<p_{0}$ ), но число вкрапляемых символов $k$ в этом случае имеет среднее $\mathbf{E}_{\theta} k=n \theta=o(\sqrt{n})$, что может быть недостаточным;

(2) $\Delta=\Delta_{\theta} \rightarrow \infty$, то есть $p_{1}=p_{1}(n) \rightarrow p_{0}$, тогда для $\theta=\theta_{n}$ получаем условие $\theta_{n}=o\left(1 / \sqrt{n \Delta_{n}}\right)$, и для числа вкрапляемых символов возможности расширяются в среднем до границы $n \theta_{n}=o\left(\sqrt{n} / \Delta_{n}\right)$, например, при $\Delta_{n} \sim 1 / \sqrt{n}$ эта граница имеет порядок $o(n)$.

Если же условие (10) не выполняется, то при $\sqrt{\theta} \Delta \rightarrow t>0$ построенный критерий различает гипотезы $H_{0}$ и $H_{\theta}$ с ошибками, асимптотически равными $\alpha$ и $1-\Phi\left(t / \sqrt{p_{0} q_{0}}=\zeta_{\alpha}\right)$, а при $\sqrt{n} \theta \Delta \rightarrow \infty$ он состоятелен (если верна альтернатива $H_{\theta}$, то этот факт обнаруживается критерием с вероятностью, стремящейся к единице при $n \rightarrow \infty)$.

\section{4. Оценивание параметра $\theta$}

Если в результате тестирования принимается гипотеза $H_{1}$ о наличии вкраплений, то желательно иметь информацию о величине интенсивности вкраплений, что требует построения приемлемых оценок для этого параметра модели. Прежде всего рассмотрим вопрос о существовании несмещенной оценки для $\theta$. Из теории полных достаточных статистик (см., например, раздел 2.3.2 в [1]) следует, что в нашем случае несмещенная оценка, если она существует, - это такая функция $\psi\left(\rho_{n}\right)$ от статистики $\rho_{n}$, которая удовлетворяет уравнению несмещенности

$$
\mathbf{E}_{\theta} \psi\left(\rho_{n}\right)=\theta
$$

для всех $\theta$. Из (1) и (3) следует, что

$$
\mathbf{E}_{\theta} \rho_{n}=n p_{\theta}=n\left(h_{0}-\theta \Delta\right)
$$

или

$$
\mathbf{E}_{\theta} \frac{n p_{0}-\rho_{n}}{n \Delta}=0 .
$$


Следовательно, уравнению (11) удовлетворяет функция

$$
\psi\left(\rho_{n}\right)=\frac{n p_{0}-\rho_{n}}{n \Delta}
$$

и решение единственно.

Чтобы принять статистику (13) в качестве разумной оценки для $\theta$, необходимо, чтобы ее возможные значения удовлетворяли условию $0 \leqslant \psi\left(\rho_{n}\right) \leqslant 1$ (множество значений оценки и оцениваемого параметра должны совпадать). Решая неравенство $\psi\left(\rho_{n}\right) \geqslant 0$, находим, что $\rho_{n} \geqslant n p_{0}$. Из другого неравенства $\psi\left(\rho_{n}\right) \leqslant 1$ легко получить, что $\rho_{n} \geqslant n p_{1}$.

Итак, необходимому условию $\psi\left(\rho_{n}\right) \in[0,1]$ удовлетворяют лишь значения статистики $\rho_{n}$ в интервале $\left[n p_{1}, n p_{0}\right]$. При $\rho_{n}<n p_{1}$ статистика $\psi\left(\rho_{n}\right)>1$, а при $\rho_{n}>n p_{0}$ статистика $\psi\left(\rho_{n}\right)<0$. Таким образом при $\rho_{n} \notin\left[n p_{1}, n p_{0}\right]$ статистика $\psi\left(\rho_{n}\right)$ не может быть использована в качестве оценки параметра $\theta$. Следовательно, в нашей модели несмещенной оценки для $\theta$ не существует.

Базируясь на статистике (13), можно предложить следующую естественную оценку $\theta$ :

$$
\hat{\theta}_{n}= \begin{cases}1, & \text { если } \rho_{n}<n p_{1}, \\ \frac{n p_{0}-\rho_{n}}{n \Delta}, & \text { если } n-p_{1} \leqslant \rho_{n} \leqslant n p_{0}, \\ 0, & \text { если } \rho_{n}>n p_{0} .\end{cases}
$$

Дополнительным обоснованием оценки $\hat{\theta}$ является тот факт, что она совпадает с оценкой максимального правдоподобия (ОМП), то есть она максимизирует функцию правдоподобия $L_{\theta}$. Действительно, дифференцируя логарифм функции правдоподобия

$$
l(\theta)=\ln L_{\theta}=\rho_{n} \ln \left(p_{0}-\theta \Delta\right)+\left(n-\rho_{n}\right) \ln \left(q_{0}+\theta \Delta\right),
$$

находим, что

$$
l^{\prime}(\theta)=-\frac{\rho_{n} \Delta}{p_{0}-\theta \Delta}+\frac{\left.n p_{0}-\rho_{n}-n\right) \Delta}{q_{0}+\theta \Delta}=\Delta \frac{n p_{0}-\rho_{n}-n \Delta \theta}{p_{\theta} q_{\theta}} .
$$

Если $\rho_{n}<n p_{1}$, то $n p_{0}-\rho_{n}-n \Delta \theta>n \Delta(1-\theta)>0$ при $\theta<1$, то есть функция $l(\theta)$ монотонно возрастает и достигает своего максимума в граничной точке $\theta=1$.

Если $\rho_{n}>n p_{0}$, то $n p_{0}-\rho_{n}-n \Delta \theta<-n \Delta(1-\theta)<0$, то есть, функция $l(\theta)$ монотонно убывает и потому имеет максимум в граничной точке $\theta=0$.

Наконец, если $\rho_{n}$ удовлетворяет условию $n p_{1} \leqslant \rho_{n} \leqslant n p_{0}$, то $l^{\prime}(\theta)=0$ для $\theta=\left(n p_{0}-\rho_{n}\right) /(n \Delta)$ и в этой точке $l^{\prime \prime}(\theta)<0$, то есть это есть точка максимума функции $l(\theta)$.

Таким образом, в нашей модели принцип максимального правдоподобия приводит к единственному решению, и ОМП имеет указанный в (14) вид.

Обсудим теперь вопрос о построении доверительного интервала для параметра $\theta$. Поскольку параметр $\theta$ в нашем случае является аргументом параметра $p_{\theta}$ биномиального распределения (2), доверительный интервал для $\theta$ можно рассчитать, используя известные методы расчета доверительного интервала для параметра $p$ биномиального распределения $\operatorname{Bi}(n, p)$. А именно, если для доверительного уровня $\gamma$ рассчитан доверительный интервал $\left(p_{H}, p_{B}\right)$, то решая неравенство $p_{h} \leqslant p_{\theta} \leqslant p_{B}$ с учетом (1) относительно $\theta$, получаем $\gamma$-доверительный интервал для $\theta$ вида

$$
\frac{p_{0}-p_{\theta}}{\Delta}<\theta<\frac{p_{0}-p_{H}}{\Delta} .
$$


Наконец, рассмотрим еще вопрос об асимптотических свойствах оценки $\hat{\theta}_{n}$ для больших выборок (при $n \rightarrow \infty)$ ). Из асимптотической нормальности (7) статистики $\rho_{n}$ следует, что события $\left\{\rho_{n}<n p_{1}\right\}$ и $\left\{\rho_{n}<n p_{0}\right\}$ имеют вероятности, стремящиеся к нулю при $n \rightarrow \infty$, следовательно, с вероятностью, стремящейся к единице, оценка $\hat{\theta}_{n}$ имеет вид $\left(n p_{0}-\rho_{n}\right) /(n \Delta)$, и эта оценка асимптотически нормальна со средним $\theta$ и дисперсией $p_{\theta} q_{\theta} /\left(n \Delta^{2}\right)$, стремящейся к нулю 0 при $n \rightarrow \infty$. Таким образом, оценка $\hat{\theta}_{n}$ является асимптотически несмещенной и состоятельной, что позволяет рассчитать асимптотический $\gamma$-доверительный интервал для параметра $\theta$ :

$$
\hat{\theta}_{n}-\frac{c_{\gamma}}{\Delta} \sqrt{p_{\hat{\theta}} q_{\hat{\theta}} / n} \leqslant \theta \leqslant \hat{\theta}_{n}+\frac{c_{\gamma}}{\Delta} \sqrt{p_{\hat{\theta}} q_{\hat{\theta}} / n}
$$

где

$$
c_{\gamma}=\Phi^{-1}\left(\frac{1+\gamma}{2}\right)
$$

Сформулируем асимптотический вариант оценивания в виде следующего итогового утверждения.

Теорема 2. Пусть $\sqrt{n} \Delta \rightarrow \infty$, тогда принимается гипотеза о наличии вкраплений $H_{1}: \theta>0$ и асимптотически несмещенной и состоятельной оценкой параметра $\theta$ является статистика

$$
\hat{\theta}_{n}=\frac{n p_{0}-\rho_{n}}{n \Delta}
$$

а асимптотический $\gamma$-доверительный интервал для $\theta$ имеет вид (15).

\section{5. Полиномиальная модель}

В этом разделе мы рассмотрим обобщение предыдущей модели вкраплений на произвольную полиномиальную модель, то есть на случай, когда последовательности $\bar{\xi}_{0}=\left(\xi_{01}, \xi_{02}, \ldots, \xi_{0 n}\right), \bar{\xi}_{1}=\left(\xi_{11}, \xi_{12}, \ldots, \xi_{1 n}\right)$ состоят из независимых случайных величин, принимающих значения из некоторого конечного алфавита $A=\left(A_{1}, A_{2}, \ldots, A_{N}\right)$, $N \geqslant 2$ (при этом механизм вкраплений $\bar{\delta}+\left(\delta_{1}, \delta_{2}, \ldots, \delta_{n}\right)$ остается прежним).

Пусть $\bar{p}_{0}=\left(p_{01} \ldots, p_{0 N}\right), \bar{p}_{1}=\left(p_{11} \ldots, p_{1 N}\right)$, где

$$
p_{0 j}=\mathbf{P}\left(\xi_{0 i}=A_{j}\right), \quad p_{1 j}=\mathbf{P}\left(\xi_{1 i}=A_{j}\right), \quad j=1, \ldots, N, \quad i \geqslant 1 .
$$

Тогда наблюдаемая последовательность $\bar{\xi}=\left(\xi_{1}, \ldots, \xi_{n}\right)$, где

$$
\xi_{i}=I(\delta=0) \xi_{01}+I(\delta=1) \xi_{1 j}, \quad i=1, \ldots, n,
$$

также является полиномиальной, для которой

$$
p_{\theta j}=\mathbf{P}\left(\xi=A_{j}\right)-(1-\theta) p_{0 j}+\theta p_{1 j}=p_{o j}-\theta \Delta_{j},
$$

где

$$
\Delta_{j}=p_{0 j}-p_{1 j}, \quad j=1, \ldots, N, \quad i \geqslant 1 .
$$


В данном случае функция правдоподобия есть

$$
L(\bar{\xi})=\prod_{i=1}^{n} \prod_{j=1}^{N} p_{\theta j}^{I\left(\xi_{i}=A_{j}\right)}=\prod_{j=1}^{N} p_{\theta j}^{v_{n j}}
$$

где

$$
v_{n j}=\sum_{i=1}^{n} I\left(\xi_{i}-A_{j}\right)
$$

есть частота появления символа $A_{j}$ в последовательности $\bar{\xi}$. Тем самым достаточной статистикой в данной модели является вектор частот $\bar{v}=\left(v_{n 1}, x_{n 2}, \ldots, v_{n N}\right)$, имеющий полиномиальное распределение

$$
\mathscr{L}_{\theta}(\bar{v})=M\left(n ; \bar{p}_{\theta}=\left(p_{\theta 1}, \ldots, p_{\theta N}\right)\right) .
$$

Из теории полиномиального распределения известно (см., например, разделы 2.4.4 и 4.2.2 в [1]), что при $n \rightarrow \infty$ вектор частот $\bar{v}_{n}$ асимптотически нормален с параметрами $n \bar{p}_{\theta}$ и $n \Sigma(\theta)$, где $\Sigma(\theta)=\left(\sigma_{i j}(\theta)=p_{\theta i}\left(\delta_{i j}-p_{\theta j}\right)\right), \delta_{i j}-$ символ Кронекера, и статистика хи-квадрат

$$
X_{n}^{2}=\sum_{j=1}^{N} \frac{\left(v_{n j}-n p_{0 j}\right)^{2}}{n p_{0 j}}
$$

имеет при справедливости гипотезы $H_{0}: \theta=0$ предельное при $n \rightarrow \infty$ распределение $\chi^{2}(N-1)$.

Более того, при любой альтернативе, задаваемой вектором $\bar{p}=\left(p_{1}, \ldots, p_{N}\right)$ вероятностей появления знаков алфавита $A$ на каждом месте наблюдаемой последовательности $\bar{\xi}$,

$$
\mathbf{E}\left(X_{n}^{2} \mid \bar{p}\right)=n \sum_{j=1}^{N} \frac{p_{j}-p_{0 j}^{2}}{p_{0 j}}+\sum_{j=1}^{N} \frac{\left(p_{j}\left(1-p_{j}\right)\right.}{p_{0 j}},
$$

то есть в нашем случае (при $\bar{p}=\bar{p}_{\theta}$ )

$$
\mathbf{E}_{\theta} X_{n}^{2}=n \theta^{2} \sum_{j=1}^{N} \frac{\Delta_{j}^{2}}{p_{0 j}} \sum_{j=1}^{N} \frac{\left(p_{0 j}-\theta \Delta_{j}\right)\left(1-p_{0 j}+\theta \Delta_{j}\right)}{p_{0 j}} .
$$

Отсюда следует, что если при $n \rightarrow \infty$ выполняется условие

$$
\theta \Delta \rightarrow 0, \quad \Delta=\max _{1 \leqslant j \leqslant N}\left|\Delta_{j}\right|,
$$

To

$$
\mathbf{E}_{\theta} X_{n}^{2}=N-1+\lambda_{n}^{2}+o(1)
$$

где

$$
\lambda_{n}^{2}=n \theta^{2} \sum_{j=1}^{N} \frac{\Delta_{j}^{2}}{p_{0 j}} .
$$

Далее, если близкая альтернатива задается вектором $\bar{p}=\bar{p}(n)$ вида

$$
\bar{p}(n)=\bar{p}_{0}+\frac{\bar{\beta}}{\sqrt{n}},
$$


где $\bar{\beta}=\left(\beta_{1}, \ldots, \beta_{N}\right) \neq \overline{0}-$ фиксированный вектор, задающий отклонение от гипотезы $H_{0}\left(\sum_{j=1}^{N} \beta_{j}=0\right)$, то

$$
\mathscr{L}\left(X_{n}^{2} \mid \bar{p}(n)\right) \rightarrow \chi^{2}\left(N-1 ; \lambda^{2}\right)
$$

при $n \rightarrow \infty$, где $\chi^{2}\left(N-1 ; \lambda^{2}\right)-$ нецентральное $\chi^{2}$ распределение с $N-1$ степенями свободы и параметром нецентральности

$$
\lambda^{2}=\sum_{j=1}^{N} \frac{\beta_{j}^{2}}{p_{0 j}} .
$$

В нашем случае альтернативы к нулевой гипотезе $H_{0}: \theta=0$, то есть, к гипотезе об отсутствии вкраплений, задаются вектором

$$
\bar{p}_{\theta}=\left(p_{\theta 1}, \ldots, p_{\theta N}\right)=p_{0}-\theta \bar{\Delta}, \quad \bar{\Delta}=\left(\Delta_{1}, \ldots, \Delta_{N}\right)=\bar{p}_{0}-\bar{p}_{1}
$$

(в этом случае мы будем говорить об альтернативе $H_{\theta}$ ), поэтому (18) будет иметь место, если при $n \rightarrow \infty$,

$$
-\sqrt{n} \theta \bar{\Delta}=\bar{\beta}+o(1)
$$

В этом случае будет также выполнено условие (16) и величина $\lambda_{n}^{2}$ в (17) будет иметь предел (19) при $n \rightarrow \infty$.

В итоге мы получаем следующее утверждение.

Теорема 3. Если в полиномиальной модели вкрапления близкие к гипотезе $H_{0}: \theta=0$ альтернативы $H_{\theta}$ задаются условием (20), то при $n \rightarrow \infty$

$$
\mathbf{E}_{\theta} X_{n}^{2}=N-1+\lambda^{2}
$$

и более того,

$$
\mathscr{L}_{\theta}\left(X_{n}^{2}\right) \rightarrow \chi^{2}\left(N-1 ; \lambda^{2}\right),
$$

где параметр нецентральности $\lambda^{2}$ указан в (19).

Этот результат дает возможность дать ответ на вопрос о том, как следует организовать процесс вкрапления, чтобы гипотезы $H_{0}: \theta=0$ (отсутствие вкраплений) и $H_{1}: \theta>0$ (наличие вкраплений) были статистически неразличимы, когда наблюдается длинная $(n \rightarrow \infty)$ последовательность $\bar{\xi}=\left(\xi_{1}, \ldots, \xi_{n}\right), \xi_{i} \in A, i=1, \ldots, n$.

Именно, гипотезы $H_{0}$ и $H_{1}$ будут асимптотически неразличимы, если в рассматриваемой модели параметры $\theta=\theta(n)$ и $\bar{p}_{1}=\bar{p}_{1}(n)=\bar{p}_{0}-\bar{\Delta}(n)$ удовлетворяют при $n \rightarrow \infty$ условию

$$
\sqrt{n} \theta(n) \bar{\Delta}(n) \rightarrow 0
$$

(в этом случае тестовая статистика $X_{n}^{2}$ имеет одно и тоже предельное распределение $\chi^{2}(N-1)$ при нулевой гипотезе $H_{0}$ и при альтернативной гипотезе $H_{1}$.

Если выполняется условие $(20)$, то в задаче $\left(H_{0}, H_{1}\right)$ критерий $\chi^{2}$, задаваемый критическим множеством

$$
X_{1 \alpha}=\left\{X_{n}^{2}>\chi_{1-\alpha, N-1}^{2}\right\}
$$


(здесь $\chi_{1-\alpha, N-1}^{2}$ есть $(1-\alpha)$-квантиль распределения $\left.\chi^{2}(N-1)\right)$ будет различать гипотезы $H_{0}$ и $H_{1}$ с ошибками асимптотически равными $\alpha$ (ошибка первого рода) и $F_{N-1}\left(\chi_{1-\alpha, N-1}^{2} ; \lambda^{2}\right)$ (ошибка второго рода), где $F_{N_{1}}\left(t ; \lambda^{2}\right)-$ функция распределения случайной величины $\chi^{2}\left(N-1 ; \lambda^{2}\right)$ и $\lambda^{2}$ приведено в (19).

Наконец, если величина $\lambda_{n}^{2} \rightarrow \infty$, то против таких далеких альтернатив $H_{\theta}$ критерий $\chi^{2}$ состоятелен.

Таким образом, при полиномиальной модели основной вывод оказался вполне аналогичным соответствующему заключению для бернуллиевской модели (ср. условия (21) и $(10))$.

\section{Список литературы}

1. Ивченко Г. И., Медведев Ю. И., Математическая статистика. Высшая школа, Москва, 1990.

2. Пономарев К. И., Об одной статистической модели стеганографии. Дискретная математика (2009) 21, №2, 138-145.

Статья поступила 8.12.2008. 